\title{
ERRATUM
}

\section{Human Immunodeficiency Virus Type 1 Resistance or Cross-Resistance to Nonnucleoside Reverse Transcriptase Inhibitors Currently under Development as Microbicides}

Philippe Selhorst, Ana C. Vazquez, Katty Terrazas-Aranda, Johan Michiels, Katleen Vereecken, Leo Heyndrickx, Jan Weber, Miguel E. Quiñones-Mateu, Kevin K. Ariën, and Guido Vanham

Virology Unit, Institute of Tropical Medicine, Antwerp, Belgium; Diagnostic Hybrids, Inc., Quidel Company, HIV Program,Cleveland, Ohio; Department of Biomedical Sciences, University of Antwerp, Antwerp, Belgium; and Faculty of Medicine and Pharmacy, Free University of Brussels, Brussels, Belgium

Volume 55, no. 4, p. 1403-1413, 2011. Page 1411: The first sentence of the Acknowledgments should read "We are grateful to Bo Öberg for his generous gift of reagents and for the proofreading of the manuscript and to Bertrand Dirié, Jurgen Joossens, and Koen Augustyns (University Antwerp Medicinal Chemistry) for chemical synthesis of lersivirine.” 